\title{
Erratum to: 18F-FDG PET/MRI evaluation of retroperitoneal fibrosis: a simultaneous multiparametric approach for diagnosing active disease
}

\author{
Verena Ruhlmann ${ }^{1}$ - Thorsten Dirk Poeppel ${ }^{1}$ • Alexander Sascha Brandt ${ }^{2}$. \\ Johannes Grueneisen ${ }^{3}$. Marcus Ruhlmann ${ }^{1}$ - Jens Matthias Theysohn ${ }^{3}$. \\ Michael Forsting ${ }^{3} \cdot$ Andreas Bockisch $^{1} \cdot$ Lale Umutlu $^{3}$
}

Published online: 22 March 2016

(C) Springer-Verlag Berlin Heidelberg 2016

Erratum to: European Journal of Nuclear Medicine and Molecular Imaging

DOI 10.1007/s00259-016-3351-3

The original version of the above article contained a mistake in the name of Johannes Grüneisen. It should have been Johannes Grueneisen.

The online version of the original article can be found at http://dx.doi.org/ 10.1007/s00259-016-3351-3.

\section{Verena Ruhlmann}

verena.ruhlmann@uk-essen.de

1 Department of Nuclear Medicine, Medical Faculty, University Duisburg-Essen, University Hospital Essen, Hufelandstraße 55, 45147 Essen, Germany

2 Department of Urology and Paediatric Urology, HELIOS Medical CenterWuppertal, University HospitalWitten/Herdecke, Heusnerstr. 40, 42283 Wuppertal, Germany

3 Department of Diagnostic and Interventional Radiology and Neuroradiology, University Hospital Essen, University Duisburg-Essen, Hufelandstraße 55, 45122 Essen, Germany 